\title{
Laparoscopic-Assisted Endoscopic Resection of a Gastric Leiomyoma
}

\author{
Shannon Acker ${ }^{1}$ Megan Dishop ${ }^{2}$ Gregory Kobak ${ }^{3}$ \\ 1 Department of General Surgery, University of Colorado, Aurora, \\ Colorado, United States \\ 2 Department of Pediatric Pathology, University of Colorado, Aurora, \\ Colorado, United States \\ ${ }^{3}$ Department of Pediatric Gastroenterology, University of Colorado, \\ Aurora, Colorado, United States \\ ${ }^{4}$ Department of Pediatric Surgery, University of Colorado, Aurora, \\ Colorado, United States
}

Eur J Pediatr Surg Rep 2014;2:3-6.
Padade Vue ${ }^{3} \quad$ Stig Somme 4

\author{
Address for correspondence Shannon Acker, MD, Department of \\ General Surgery, University of Colorado, $12631 \mathrm{E}, 17$ th Avenue C302, \\ Aurora, Colorado 80045, United States \\ (e-mail: shannon.acker@ucdenver.edu).
}

\begin{abstract}
Keywords

- gastric leiomyoma

- CD117

- endoscopy

- DOG1

Leiomyomas are infrequent benign intestinal tumors that can arise at any age and location within the gastrointestinal (GI) tract. These tumors can cause symptoms including abdominal pain, obstruction, intussusception, volvulus, GI bleeding, or a mass and should be resected if symptomatic. Open surgical resection is considered the standard for removing these tumors. However, recent improvements in endoscopic and laparoscopic equipment have made it possible to utilize minimally invasive techniques of tumor removal including complete endoscopic resection or endoscopic-assisted laparoscopic resection. We present the case of an adolescent female with a large mass located at the gastroesophageal junction (GEJ) causing GI bleeding. Given the location of the mass near the GEJ and the morbidity associated with surgical resection, we performed laparoscopic-assisted complete endoscopic resection of tumor. In addition, this tumor had an unusual immunohistochemical-staining pattern, with focal expression of markers more often seen in GI stromal tumors, elucidating a gray area between these two tumor classes with potential implications for patient follow-up. Laparoscopicassisted endoscopic resection of benign tumors is a useful technique that can be employed to facilitate resection of mucosal and subserosal masses near the GEJ with minimal morbidity.
\end{abstract}

\section{Introduction}

Leiomyomas are rare tumors that can arise anywhere in the gastrointestinal (GI) tract although they occur most frequently in the stomach, jejunum, or ileum. ${ }^{1}$ These tumors are quite rare in children; only case reports or short case series are found in the literature. ${ }^{2-4}$ These tumors are often asymptomatic but can also present with an abdominal mass, obstruction, intussusception, volvulus, GI bleeding, or abdominal pain and should be resected if symptomatic. Here, we present the rare case of a 16-year-old female patient with a gastric leiomyoma with an unusual immunohistochemical staining pattern. We used a method of laparoscopic-assisted endoscopic resection to completely excise the mass.

\section{Case Report}

A 16-year-old previously healthy female patient (weight, $67.5 \mathrm{~kg}$; height, $172 \mathrm{~cm}$ ) presented to the emergency department after experiencing dizziness followed by syncope. Upon received

October 16, 2013

accepted after revision

January 3, 2013

published online

March 12, 2014

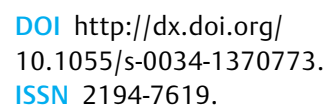

(c) 2014 Georg Thieme Verlag KG
Stuttgart · New York

License terms

(ㄷ) (1) $\ominus$ (2) 
arrival, she had an episode of hematemesis. Further questioning revealed that the patient had two episodes of melena 24 hours before presentation. The patient was normotensive with a normal heart rate. Physical examination was unremarkable. Laboratory studies obtained in the emergency department were remarkable for hemoglobin of $7.4 \mathrm{~g} / \mathrm{dL}$. She was seen by the gastroenterology service and underwent nasogastric lavage, which was notable for blood clots. The patient was transfused one unit of packed red blood cells and admitted for further work up.

On hospital day number 1 , she underwent upper intestinal endoscopy; a large gastric polypoid lesion was identified in the fundus/cardia region of the stomach. The polyp was approximately $2 \times 3 \mathrm{~cm}$ and had a small $3 \mathrm{~mm}$ ulceration without evidence of active bleeding. A superficial biopsy of the polyp was obtained endoscopically, however, this was found to contain only normal mucosa on permanent section. The mass was very close to the gastroesophageal junction (GEJ), making surgical excision difficult, likely requiring excision of the GEJ. Our team was faced with the dilemma of how to approach diagnosis and resection for this patient. Given the possibility of benign pathology with the large morbidity of GEJ resection, we devised a plan to proceed with laparoscopic-assisted endoscopic resection of the polyp. With this approach, we felt we would be able to obtain a tissue diagnosis and resect the mass with minimum morbidity, while also acknowledging the risk of incomplete mass excision and need for second procedure pending pathology results. For this reason, after consultation with the family, she was scheduled for a laparoscopic-assisted endoscopic resection of the polyp approximately 2 weeks after her initial presentation.

The procedure began with laparoscopic exploration of the GEJ and localization of the mass (camera, $5 \mathrm{~mm} 30$ degree Karl Storz HD, Karl Storz, Tuttlingen, Germany, placed at the umbilicus). Two 5-mm ports (Mini Step trocars, Covidien plc, Dublin, Ireland) and one liver retractor (articulating circle retractor, $5 \mathrm{~mm}, 40 \mathrm{~cm}$, Snowden-Pencer, Waukegan, Illinois, United States) were placed to visualize the proximal stomach. The liver retractor was placed through a port in the right upper quadrant. The mass was palpated with the laparoscopic instruments and was felt to be approximately $3 \mathrm{~cm}$ in diameter directly adjacent to the GEJ. The endoscope was then inserted and the mass was visualized near the GEJ with possible extension into the lower esophageal mucosa (-Fig. 1). The mass was successfully removed endoscopically using electrocautery and enucleation, utilizing the laparoscopic instruments to provide counter pressure and traction. Given the location of the tumor so close to the GEJ, the laparoscopic instruments were critical to help position the mass for optimal endoscopic resection. The additional benefit of the laparoscopic assistance was real-time monitoring for evidence of gastric perforation. No gastric perforation occurred. Despite the technical difficulty of the procedure given the location of the mass adjacent to the GEJ, the mass appeared to be completely resected endoscopically. The area was then fulgurated to remove any remaining tumor. The patient's postoperative course was uncomplicated.

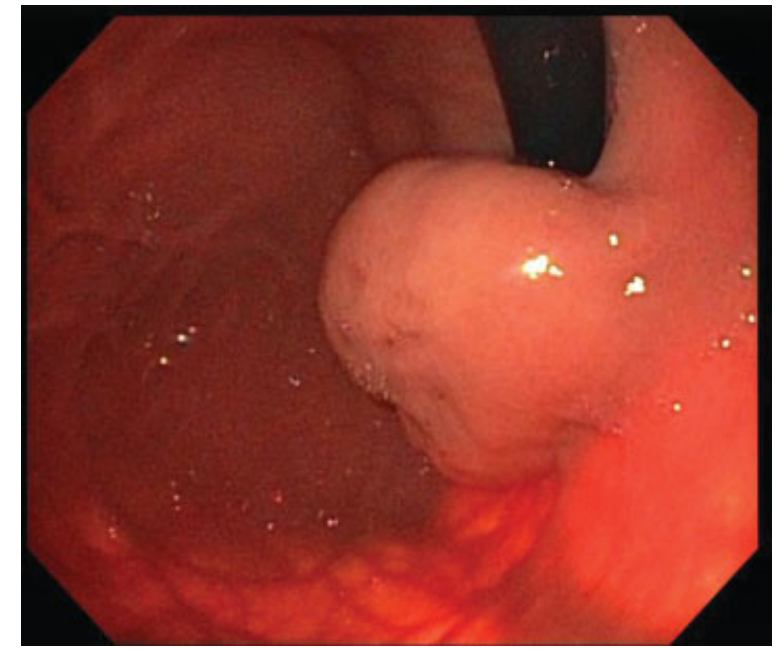

Fig. 1 Photograph taken at the time of endoscopic tumor resection. This image demonstrates the relatively large mass located right at the gastroesophageal junction.

Pathologic evaluation of the specimen showed that the polyp had very low cellularity, with morphologic, immunohistochemical, and ultrastructural features supporting smooth muscle differentiation (-Fig. 2, hematoxylin and eosin stain). There was diffuse strong positivity for smooth muscle actin (SMA) and desmin, both consistent with benign gastric leiomyoma. Interestingly, the sample also contained foci of cells positive for CD117 and DOG1 (discovered on gastrointestinal stromal tumor 1 [GIST-1]), both markers typically expressed more diffusely in gastrointestinal stromal tumors (GISTs). Pan-Cytokeratin and S100 stains were both negative. The final pathologic diagnosis was a benign leiomyoma, based on these features.

The patient underwent repeat endoscopy 6 months following resection. At this time, there was no evidence of residual tumor and only normal mucosa present on biopsy specimens.

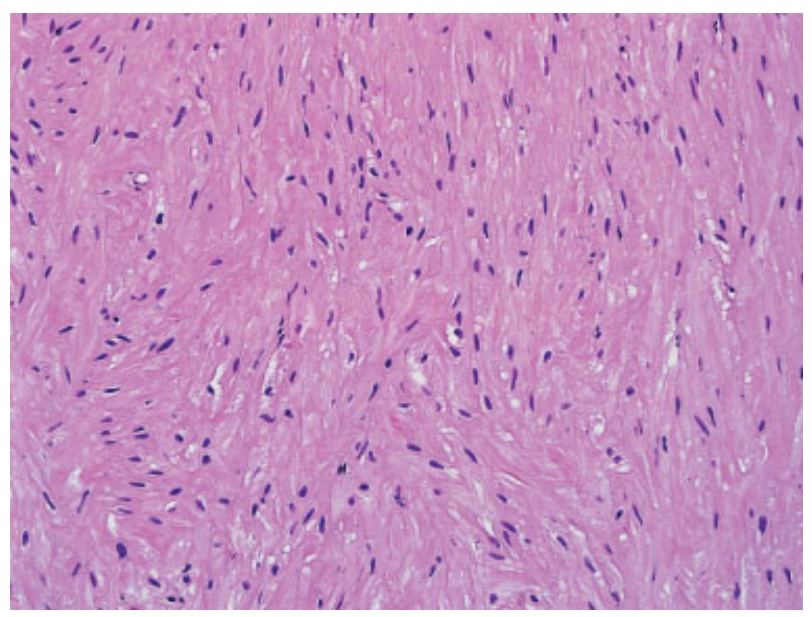

Fig. 2 Smooth muscle tumor with low cellularity. The cells form haphazard fascicles and have abundant eosinophilic cytoplasm with "cigar-shaped" nuclei. 


\section{Discussion}

We present the case of an adolescent girl with a large, symptomatic, bleeding mass near the GEJ resulting in anemia requiring blood transfusion. This unusual mass presented a management dilemma for our groups as we wanted to avoid the morbidity associated with an open resection given that this was likely a benign mass in a young patient. Collaboration between the pediatric surgeons and gastroenterologists lead to development of a plan for laparoscopic-assisted endoscopic resection of the mass. Consideration was given to the possibility of a laparoscopic wedge resection however this was not felt to be feasible given the location of the mass. Not knowing the pathologic classification of the tumor at the time of surgery further added to the importance of complete removal and development of a comprehensive and collaborative approach. Previous groups have reported the benefits of endoscopic-assisted laparoscopic resection of gastric tumors, ${ }^{5-8}$ however the previously described procedures involved a gastrotomy to ensure complete tumor resection. Our approach was unique, in that we were able to successfully excise the entire mass without creating a gastrotomy. As a result, all risks associated with surgical gastrotomy, although low, were eliminated. Other groups have previously reported success with total endoscopic resection of gastric leiomyomas. ${ }^{9,10}$ In our case, we were able to utilize a minimally invasive approach to successfully resect the mass, with the associated benefits to the patient. This technique can be used to address other benign masses located near the GEJ.

Neither the location of the tumor, the approach to resection, nor its immunohistochemical characteristics were usual in this patient. Gastric leiomyoma is a rare finding, particularly in pediatric patients, and in this case the pathologic differential diagnosis of leiomyoma versus GIST was raised. The histopathologic features including low cellularity and cytologic characteristics were most consistent with a leiomyoma, although focal expression of DOG1 and CD117 raised initial consideration of a low-grade GIST. Two immunohistochemical markers that have been used to help in making the distinction between GISTs and tumors of smooth muscle cell origin are CD117 (KIT) and DOG1. ${ }^{11,12}$ Activating mutations of KIT lead to overexpression of the KIT protein (CD117), which can be identified by immunohistochemical staining. Mutations in the KIT gene are found in up to $96 \%$ of GISTs, ${ }^{13}$ and CD117 expression is reported in 93\% of GISTs. ${ }^{14}$ DOG1 protein is expressed in 80 to $96 \%$ of GISTs, and dual expression of both CD117 and DOG1 can accurately identify 99\% of GISTs. ${ }^{13}$ Because the tumor had a histologic appearance more consistent with a smooth muscle cell tumor, diffuse strong positivity for SMA, but only focal CD117 and DOG1 expression, the tumor was determined to be a gastric leiomyoma. The immunohistochemical pattern of CD117 and DOG1 expression in this case demonstrates the overlap and potential diagnostic difficulty between these two tumor classes. This case underscores the importance of integration of all pathologic features, and that diagnosis of GIST should not be based on immunohistochemical features alone. The pattern of expression of these markers (focal vs. diffuse) should be considered in evaluating the differential diagnosis of smooth muscle tumors and GISTs.

Although this patient was diagnosed with a benign gastric leiomyoma, the overlapping immunohistochemical features with GIST raised clinical concern that she could be at increased risk of tumor recurrence or more aggressive behavior. KIT protein (CD117) is a receptor tyrosine kinase whose overexpression in GISTs leads to tumor progression and appears to drive neoplastic growth. ${ }^{15}$ In light of focal CD117 expression in this tumor, we elected to manage this patient with close follow-up to monitor for tumor recurrence. She has already undergone repeat endoscopy with no evidence of tumor recurrence 6 months following resection. Had this tumor been diagnosed as a GIST, the patient would have likely required repeat operative intervention for complete tumor excision. Although wedge or segmental resection with complete tumor removal is acceptable for GIST tumors, the rate of recurrence for tumors located at the GEJ remains high ( $42 \%$ in one series). ${ }^{16}$ For this reason, wedge resection is not recommended for tumors greater than $3 \mathrm{~cm}$ located at the GEJ, instead these patients often require laparotomy and esophageal reconstruction. ${ }^{1}$

\section{Conclusions}

Gastric leiomyoma is a rare entity in pediatric patients. We present a combined laparoscopic and endoscopic approach to tumor resection, which successfully was applied to remove all visible tumor present in the fundus/cardia of the stomach. In addition, these tumors may be difficult to distinguish from GISTs because of similar immunohistochemical markers. This patient will require close long-term follow-up to detect tumor recurrence or malignant degeneration.

Conflict of Interest

None.

\section{References}

1 Gervaz P, Huber O, Morel P. Surgical management of gastrointestinal stromal tumours. Br J Surg 2009;96(6):567-578

2 Cremin BJ. Gastric leiomyoma in a child. Br J Radiol 1969;42(495): 226

3 Ladd AP, Grosfeld JL. Gastrointestinal tumors in children and adolescents. Semin Pediatr Surg 2006;15(1):37-47

4 Miettinen M, Lasota J, Sobin LH. Gastrointestinal stromal tumors of the stomach in children and young adults: a clinicopathologic, immunohistochemical, and molecular genetic study of 44 cases with long-term follow-up and review of the literature. Am J Surg Pathol 2005;29(10):1373-1381

5 Payne WG, Murphy CG, Grossbard LJ. Combined laparoscopic and endoscopic approach to resection of gastric leiomyoma. J Laparoendosc Surg 1995;5(2):119-122

6 Walsh RM, Heniford BT. Laparoendoscopic treatment of gastric stromal tumors. Semin Laparosc Surg 2001;8(3):189-194

7 Ludwig K, Wilhelm L, Scharlau U, Amtsberg G, Bernhardt J. Laparoscopic-endoscopic rendezvous resection of gastric tumors. Surg Endosc 2002;16(11):1561-1565

8 Walsh RM, Ponsky J, Brody F, Matthews BD, Heniford BT. Combined endoscopic/laparoscopic intragastric resection of gastric stromal tumors. J Gastrointest Surg 2003;7(3):386-392 
6 Laparoscopic-Assisted Endoscopic Resection of a Gastric Leiomyoma Acker et al.

9 Kajiyama T, Hajiro K, Sakai M, et al. Endoscopic resection of gastrointestinal submucosal lesions: a comparison between strip biopsy and aspiration lumpectomy. Gastrointest Endosc 1996;44(4):404-410

10 Spinelli P, Cerrai FG, Cambareri AR, Meroni E, Pizzetti P. Two-step endoscopic resection of gastric leiomyomas. Surg Endosc 1993; 7(2):90-92

11 Kuhlgatz J, Sander B, Golas MM, et al. Differential diagnosis of gastrointestinal leiomyoma versus gastrointestinal stromal tumor. Int J Colorectal Dis 2006;21(1):84-88

12 Miettinen M, Wang ZF, Lasota J. DOG1 antibody in the differential diagnosis of gastrointestinal stromal tumors: a study of 1840 cases. Am J Surg Pathol 2009;33(9):1401-1408
13 Lopes LF, West RB, Bacchi LM, van de Rijn M, Bacchi CE. DOG1 for the diagnosis of gastrointestinal stromal tumor (GIST): Comparison between 2 different antibodies. Appl Immunohistochem Mol Morphol 2010;18(4):333-337

14 CD117 antibody panel. Path IQ Immunoquery. Available at: https://immunoquery.pathiq.com. Accessed on April 15, 2013

15 Cypriano MS, Jenkins JJ, Pappo AS, Rao BN, Daw NC. Pediatric gastrointestinal stromal tumors and leiomyosarcoma. Cancer 2004;101(1):39-50

16 An JY, Choi MG, Noh JH, et al. Gastric GIST: a single institutional retrospective experience with surgical treatment for primary disease. Eur J Surg Oncol 2007;33(8):1030-1035 